\title{
The activation of a Suv39h1-repressive antisense IncRNA by OCT4 couples the control of H3K9 methylation to pluripotency
}

\author{
Laure D. Bernard ${ }^{1,2}$, Agnès Dubois ${ }^{1}$, Victor Heurtier ${ }^{1,2}$, Almira Chervova $^{1}$, Alexandra Tachtsidi $^{1,2}$, Noa Gil ${ }^{3,4}$, Nick Owens $^{1,5}$, \\ Sandrine Vandormael-Pournin ${ }^{1}$, Igor Ulitsky ${ }^{3}$, Michel Cohen-Tannoudji ${ }^{1}$, and Pablo Navarro ${ }^{1, *}$ \\ ${ }^{1}$ Epigenomics, Proliferation, and the Identity of Cells, Department of Developmental and Stem Cell Biology, Institut Pasteur, CNRS UMR3738, 75015 Paris, France \\ ${ }^{2}$ Sorbonne Université, Collège doctoral, F-75005 Paris, France \\ ${ }^{3}$ Department of Biological Regulation, Weizmann Institute of Science, Rehovot, Israel \\ ${ }^{4}$ Current address: Friedrich Miescher Institute for Biomedical Research, Basel, Switzerland \\ ${ }^{5}$ Current address: Institute of Biomedical and Clinical Science, University of Exeter Medical School, Exeter, UK \\ *Corresponding author: pnavarro@pasteur.fr
}

\begin{abstract}
Histone H3 Lysine 9 (H3K9) methylation, a characteristic mark of heterochromatin, is progressively implemented during development to contribute to cell fate restriction as differentiation proceeds. For instance, in pluripotent mouse Embryonic Stem (ES) cells the global levels of $\mathrm{H3K9}$ methylation are rather low and increase only upon differentiation. Conversely, H3K9 methylation represents an epigenetic barrier for reprogramming somatic cells back to pluripotency. How global H3K9 methylation levels are coupled with the acquisition and loss of pluripotency remains largely unknown. Here, we identify SUV39H1, a major H3K9 di- and tri-methylase, as an indirect target of the pluripotency network of Transcription Factors (TFs). We find that pluripotency TFs, principally OCT4, activate the expression of an uncharacterized antisense long non-coding RNA to Suv39h1, which we name Suv39h1as. In turn, Suv39hlas downregulates Suv39h1 transcription in cis via a mechanism involving the modulation of the chromatin status of the locus. The targeted deletion of the Suv39h1as promoter region triggers increased SUV39H1 expression and H3K9me2 and $H 3 K 9 \mathrm{me} 3$ levels, leading to accelerated and more efficient commitment into differentiation. We report, therefore, a simple genetic circuitry coupling the global levels of $\mathrm{H3K9}$ methylation to pluripotency in mouse ES cells.
\end{abstract}

\section{Introduction}

During development, the establishment and maintenance of distinct gene expression patterns supporting the identity of each cell type are closely linked to the regulation of chromatin states ${ }^{1}$. Two broad states have been clearly and unambiguously identified: euchromatin, associated with transcriptionally active regions, and heterochromatin, associated with gene repression ${ }^{2-5}$. Two major states of heterochromatin have been traditionally considered. Facultative heterochromatin refers to a repressive chromatin environment displaying high variability across developmental stages, cell types and cell states. Indeed, silent developmental genes are usually embedded in facultative heterochromatin ${ }^{\mathbf{3 , 5}}$. In contrast, ubiquitously silent elements such as retrotransposons and pericentromeric regions are locked by constitutive heterochromatin ${ }^{\mathbf{4 , 5}}$. These two types of heterochromatin have been thought to be distinguishable by distinct molecular signatures, with facultative heterochromatin being characterized by trimethylation of histone $\mathrm{H} 3$ lysine 27 (H3K27me3) and constitutive heterochromatin by H3K9me3, among other chromatin features ${ }^{2-5}$. Nevertheless, recent data has challenged these strict definitions ${ }^{3}$. On the one hand, constitutive heterochromatin can under some circumstances be transcribed or decorated by marks previously associated with facultative heterochromatin ${ }^{6-8}$. On the other, while $\mathrm{H} 3 \mathrm{~K} 27 \mathrm{me} 3$ and $\mathrm{H} 3 \mathrm{~K} 9 \mathrm{me} 2$ were considered as major repressive mark for developmental genes, an increasing body of evidence points to $\mathrm{H} 3 \mathrm{~K} 9 \mathrm{me} 3$ as an additional mean to silence developmental regulators as their expression is definitely shut down in particular lineages?. Hence, even though the role of $\mathrm{H} 3 \mathrm{~K} 9$ methylation in genome stability is unquestionable ${ }^{10}$, its importance in gene regulatory mechanisms during development appears to be equally important. Indeed, mouse knock-out (KO) models of H3K9 histone methyltransferases (HMTs) display penetrant phenotypes, particularly during gastrulation when pluripotency is lost and major differentiation events take place ${ }^{11,12}$. Conversely, before reaching pluripotency during early mouse embryogenesis the levels of H3K9 methylation are strictly controlled; promoting their increase, for instance by overexpressing the HMT SUV39H1, leads to developmental defects at the compaction stage ${ }^{13,14}$.

While extensive research has contributed to our understanding of how the establishment and maintenance of H3K27me3 regulates developmental transitions, how the levels of $\mathrm{H} 3 \mathrm{~K} 9$ methylation are developmentally regulated is less clear. Yet, a major distinction has been identified, particularly using pluripotent cells such as mouse Embryonic Stem (ES) cells. Indeed, H3K27me3 characterizes developmental genes even before differentiation, when they are embedded in the so-called bivalent chromatin, which is simultaneously enriched for H3K27me3 and for marks of activity ${ }^{\mathbf{1 5}}$. Upon differentiation, H3K27me 3 is either consolidated or erased in a cell-type-dependent manner ${ }^{\mathbf{1 6}}$. On the contrary, H3K9 methylation is more largely controlled at the level of its abundance: during differentiation the global levels of H3K9me2 and $\mathrm{H} 3 \mathrm{~K} 9 \mathrm{me} 3$ increase drastically ${ }^{\mathbf{1 7}, 18}$. Conversely, during 


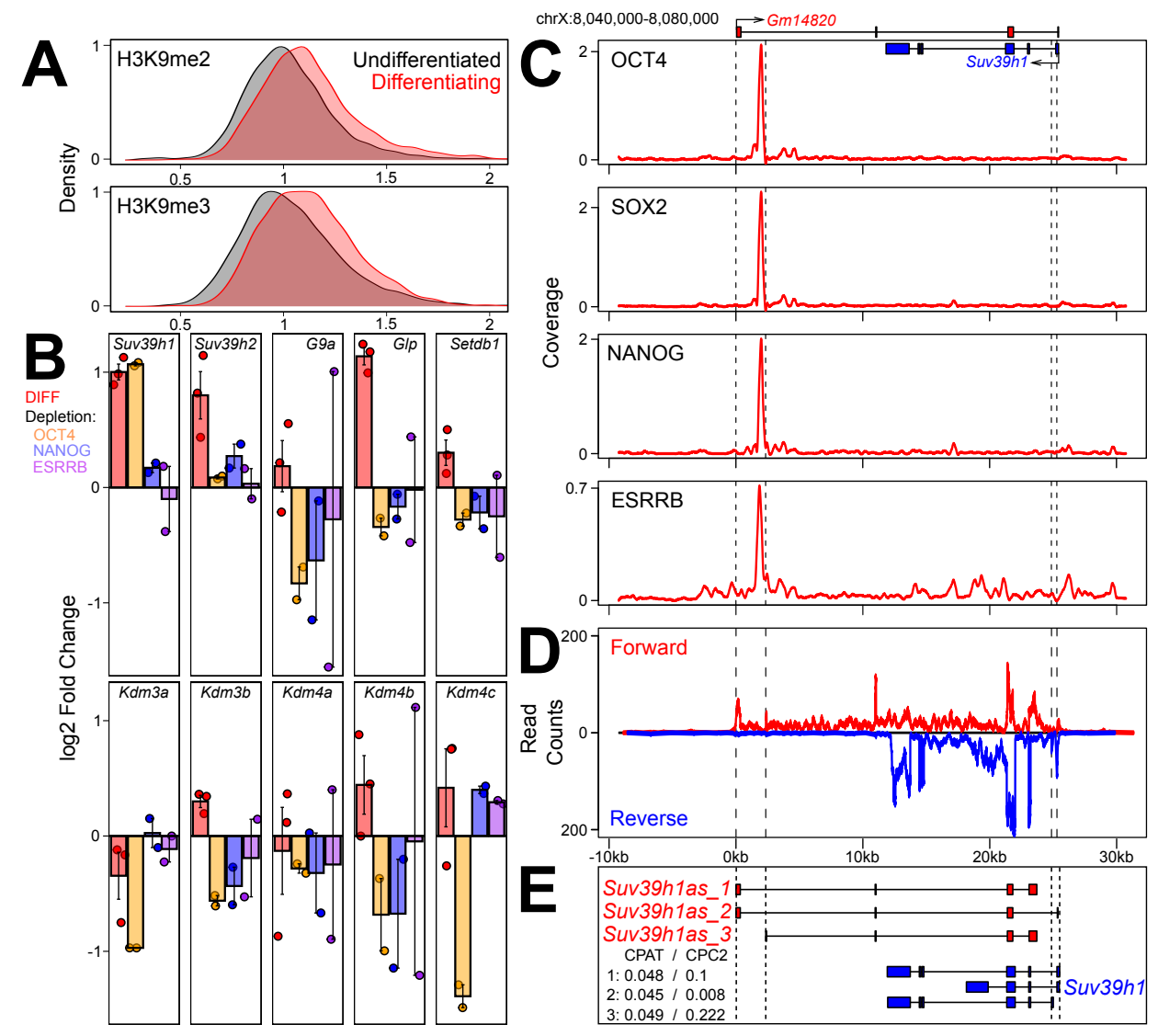

Fig. 1. Suv39h1 is downregulated by OCT4, which binds to the promoter of a Suv39h1 antisense IncRNA. (A) Distribution of H3K9me2 (top) and H3K9me3 (bottom) in undifferentiated and differentiating ES cell populations assessed by immunofluorescence (Y-axis, density; $\mathrm{X}$-axis, relative mean intensity per cell). Undifferentiated cells (black) are E14Tg2a cells cultured in FCS/LIF (black; $n=4503$ cells for H3K9me2 and 3150 for H3K9me3); differentiating cells were obtained by LIF withdrawal (red; $n=6231$ cells for H3K9me2 and 3755 cells for H3K9me3). (B) Log2 fold change of the indicated gene after differentiating ES cells as in (A) or 24h after inducing the depletion of individual TFs (OCT4, NANOG or ESRRB, as indicated) using Dox-inducible knock-out cells. Each dot represents an independent replicate and the bar and error bars the corresponding means and standard errors. (C) Average binding profile of OCT4, SOX2, NANOG and ESRRB (reads per million) across the Suv39h1/Gm14820 locus (mm10, chrX:8,040,000-8,080,000 - $40 \mathrm{~kb}$ ). Suv39h1 and Gm14820 are schematically represented on top. (D) RNA-seq profile across the Suv39h1/Gm14820 locus, with forward and reverse fragment counts expressed with positive and negative values. (E) Schematic representation of Gm14820/Suv39h1as (red) and Suv39h1 (blue) isoforms. The coding probabilities calculated with CPAT and CPC2 algorithms are shown for the three isoforms of Suv39h1as. The vertical dashed lines in (C), (D) and (E) mark the position of Suv39h1as or Suv39h1 promoters.

the induction of pluripotency in vitro through reprogramming processes, H3K9 methylation has been shown to act as a major epigenetic barrier that is in part overcome by globally reducing its levels ${ }^{\mathbf{1 8 , 1 9}}$. Therefore, while H3K27 methylation is mainly controlled by altering its genomic distribution, the global levels of $\mathrm{H} 3 \mathrm{~K} 9$ methylation display correlated changes to the differentiation status. Beyond the role of H3K9 methylation to stabilise somatic cell identities ${ }^{\mathbf{2 0}}$, how its global levels are seemingly coupled to the acquisition and loss of pluripotency, and what consequences this coupling has, remain open questions.

In this study, we aimed at understanding the molecular basis of the link between H3K9 methylation and pluripotency. We find Suv39hl to be the only HMT tightly connected to the network of transcription factors (TFs) supporting pluripotency, particularly to its main player Oct4. The analysis of the mechanisms of Suv39h1 repression by OCT4 led us to the identification of a repressive antisense long noncoding RNA (lncRNA ${ }^{\mathbf{2 0}}$ ) to the Suv39hl gene. Our work identifies a simple genetic network based on the activation of the Suv39h1 antisense by OCT4 which, in turn, represses Suv39h1 transcription thereby coupling H3K9 methylation to pluripotency. Using CRISPR-Cas9 mediated deletion of the antisense promoters, we show that its activity controls the timing and efficiency of ES cell commitment into differentiation. Our results therefore provide a mechanistic perspective into how the global levels of $\mathrm{H} 3 \mathrm{~K} 9$ methylation are regulated at the onset of differentiation to irreversibly exit pluripotency.

\section{Results}

\section{Suv39h1 expression is under the control of OCT4 in ES cells.}

Using immunofluorescence, we first confirmed that differentiation of ES cells by LIF withdrawal leads to an increase of both H3K9me2 and H3K9me3 (Fig.1A; p $<10^{-15}$ ), as expected. We hypothesized that one or several histone methyltransferases or lysine demethylases (HMTs and KDMs, respectively) ${ }^{\mathbf{2}, \mathbf{4}}$ could be differentially expressed upon differentiation, linking the loss of pluripotency to increased H3K9 methylation. To assess this, we monitored mRNA levels of HMTs and KDMs using published RNA-seq datasets ${ }^{22}$ (Fig.1B; Table S1). We found three HMTs to be upregulated upon differentiation: Suv39hl ( $\mathrm{p}=0.004)$, Suv39h2 ( $\mathrm{p}=0.044)$ and $G l p(\mathrm{p}=0.004)$. In contrast, all tested KDM displayed minor changes below 2-fold. We reasoned that the increase of Suv39h1, Suv39h2 and Glp expression could either be due to a direct control of their transcription by pluripotency TFs or to alternative mechanisms. To address this, we assessed the impact of the loss of individual pluripotency TFs (Oct4, Nanog and Esrrb) using available dox-inducible knock-outs $^{\text {23-25 }}$ (Fig.S1A). Only one, Suv39h1, was found upregulated $24 \mathrm{~h}$ after inducing the loss of pluripotency TFs, particularly of OCT4, which depletion leads to a 2-fold increase in Suv39h1 mRNA levels (Fig.1B; Table S1). Hence, after confirming Suv39hl expression changes by RT-qPCR (Fig.S1B), we hypothesized that OCT4 may act as a repressor of Suv39hl expression to maintain low levels of H3K9 methylation until the onset of differentiation. Exploration of 

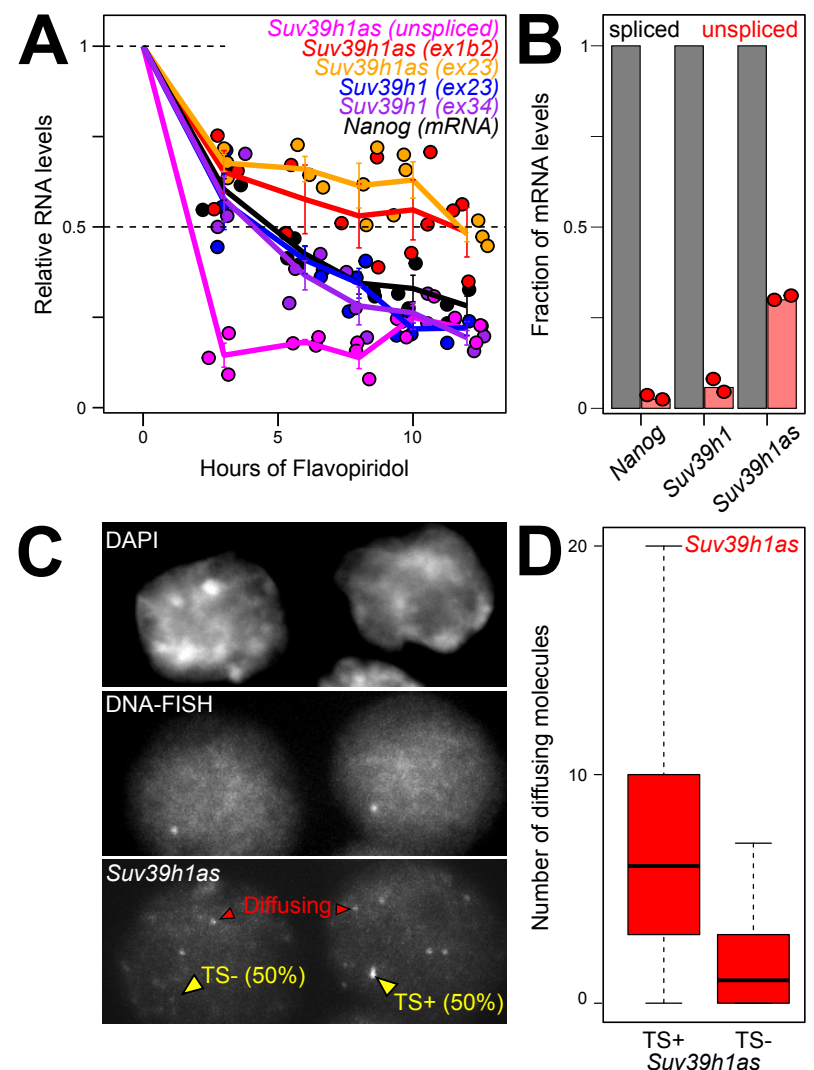

Fig. 2. Suv39h1as is a nuclear, stable and lowly expressed IncRNA. (A) RTqPCR analysis of the half-life of several RNA species during a transcription inhibition assay with Flavopiridol (X-axis, hours of treatment): Suv39h1 mRNA, using two trans-exonic primer pairs between exons 2 and 3 (blue - ex23) or exons 3 and 4 (purple - ex34); Suv39h1as, using two trans-exonic primer pairs between exons $1 \mathrm{~b}$ and 2 (red - ex1b2) or exons 2 and 3 (orange - ex23) or primer pairs amplifying the unspliced RNA (magenta - pre-mRNA); Nanog mRNA (black). Each dot represents an independent replicate and the line the corresponding mean and standard error. Ribosomal RNA (28S) was used for normalization. (B) Histogram representing unspliced RNA levels (red) relative to corresponding spliced RNAs (black) for Nanog, Suv39h1 and Suv39h1as, as measured by RNA-seq. Each dot represents an independent replicate and the bar the corresponding mean. (C) Representative sm-FISH followed by DNA-FISH visualizing Suv39h1as RNA molecules and the Suv39h1/Suv39h1as locus, respectively, in undifferentiated E14Tg2a cells. Red arrowheads indicate RNAs diffusing away from the locus, which is indicated by a yellow arrow. The proportion of actively transcribing cells is indicated ( $n=358$ cells). (D) Boxplots (median; 25-75\% percentiles; error bars) showing the number of Suv39h1as diffusible molecules counted in cells presenting an active (TS+) or inactive (TS-) Suv39h1as gene $(\mathrm{n}=358)$.

available ChIP-seq datasets ${ }^{\mathbf{2 6}}$ (Fig.1C) and direct validation by ChIP-qPCR (Fig.S1C) identified a hotspot of pluripotency TFs, including OCT4, in the vicinity of Suv39hl. However, this TF binding hotspot was found located 3' to Suv39hl, at around $27 \mathrm{~kb}$ of its promoter region. Notably, we noticed that this region coincides with the promoter region of an uncharacterized gene, Gm14820 (AK010638), antisense to and largely overlapping Suv39h1 (top of Fig.1C).

\section{Identification of a $S u v 39 h 1$ antisense IncRNA.}

Stranded RNA-seq confirmed Gm14820 to be expressed in ES cells, at levels comparable to Suv39hl (Fig.1D). Using de novo transcript assembly with our RNA-seq datasets, together with direct cDNA cloning, sequencing and RT-qPCR, we identified several isoforms expressed in ES cells (Fig.1E). All isoforms initiate from two distinct promoters, located in proximity to the region bound by pluripotency TFs, exhibit overlapping exons with $S u v 39 h 1$ and terminate within Suv39h1 or in the vicinity of its 5' end. Notably, Gm14820 is annotated as a lncRNA ${ }^{21}$. Accordingly, using two different algorithms $\left(\mathrm{CPAT}^{\mathbf{2 7}}\right.$ and $\mathrm{CPC}^{28}$ ), the nearly absent coding potential of all Gm14820 isoforms was confirmed (Fig.1E). Thereafter, we refer to Gm14820 as Suv39hlas. To further characterize Suv39hlas, we assessed the stability of its RNA products and found the half-life of its spliced and unspliced forms to be around $12 \mathrm{~h}$ and $1 \mathrm{~h} 30$, respectively (Fig.2A). However, Suv39hlas splicing is relatively inefficient compared to Suv39hl or another protein coding gene, Nanog (Fig.2B), as is generally the case for lncRNAs ${ }^{\mathbf{2 1}}$. Moreover, Suv39hlas was efficiently captured in poly-A selected RNA-seq, suggesting it is normally poly-adenylated (Table S1). Next, we aimed at visualizing Suv39hlas RNA molecules in single cells. For this, we designed oligonucleotides targeting Suv39hlas exons and performed strandspecific single molecule RNA-FISH (smFISH) coupled to DNA-FISH to identify the Suv39hlas/Suv39h1 locus, using a fosmid covering the whole region (Fig.2C). We observed that Suv39hlas is mainly detected as a bright point in the nucleus, likely representing actively transcribed loci as it coincides with the DNA-FISH signal. A small number of single Suv39hlas RNA molecules could also be detected diffusing in the nucleus and, more rarely, in the cytoplasm. Quantification of the smFISH/DNA-FISH suggested a transcriptional frequency of around $50 \%$ in the population, with a median of 6 freely diffusing RNAs in cells presenting a transcriptionally active locus (Fig.2D). Hence, we conclude that the pluripotency TFs bind close to the two promoters of a Suv39hl antisense lncRNA, which is mostly localised at its site of transcription, poly-adenylated and poorly spliced even though the spliced isoforms are relatively stable.

\section{Anticorrelated expression patterns of Suv39h1 and Suv39h1as.}

To investigate Suv39hlas and Suv39hl expression patterns we differentiated ES cells using three independent protocols based on LIF withdrawal, N2B27 or EpiLC-directed differentiation. These three assays showed a strong reduction of Suv39hlas expression after 3 days of differentiation, when Suv39hl expression increases (Fig.3A; p $<0.05$ (Suv39hl) and $\mathrm{p}<0.01$ (Suv39hlas) for all differentiation assays). Moreover, the depletion of OCT4 showed a rapid downregulation of Suv39hlas ( $<<10^{-3}$ at all time-points), reaching minimal levels of expression within $12 \mathrm{~h}$ and accompanied by a marked increase of Suv39hl expression ( $\mathrm{p}<0.05$ at all timepoints) that reached maximal levels after 24h (Fig.3B). Thus, Suv39h1 and Suv39hlas display anticorrelated expression levels. To explore this observation at the single cell level, we designed oligonucleotides across Suv39hl exons and introns to monitor Suv39h1/Suv39hlas expression by smFISH in parallel to DNA-FISH (Fig.3C,D). We found around $20 \%$ of cells actively transcribing both sense/antisense genes and around $30 \%$ transcribing either one or the other. Moreover, cells actively transcribing Suv39hlas displayed significantly $\left(\mathrm{p}<10^{-11}\right)$ fewer Suv39h1 mRNA molecules (Fig.3D). In 


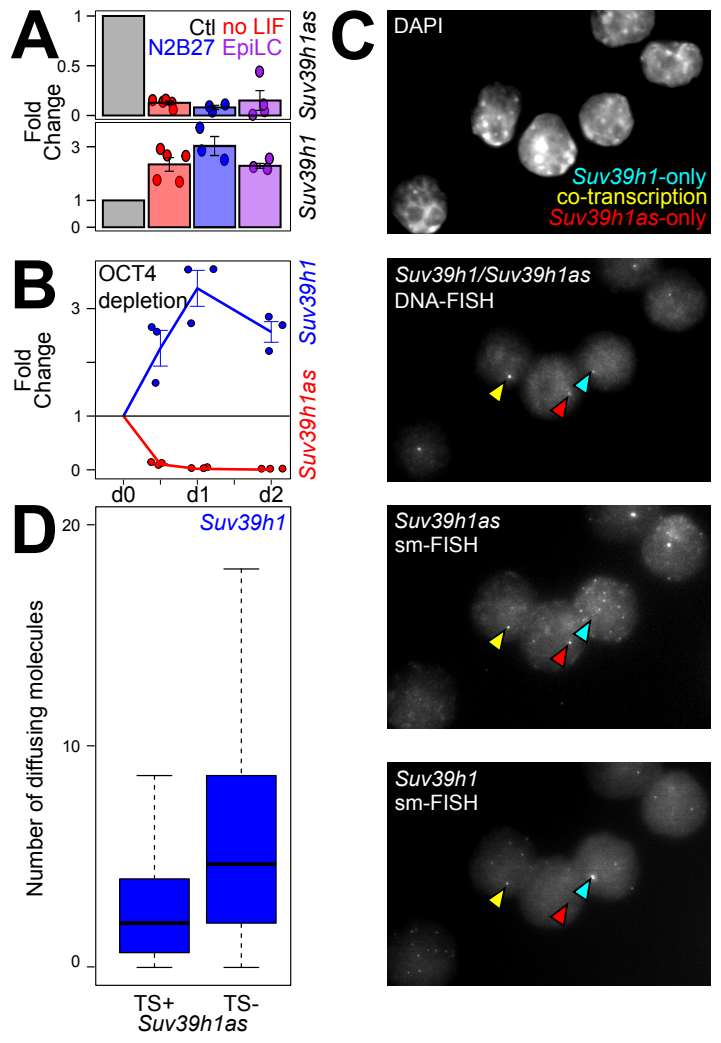

Fig. 3. Anticorrelated expression of Suv39h1 and Suv39h1as. (A) Fold change expression of Suv39h1as (top) or Suv39h1 (bottom) measured by RT-qPCR in differentiating E14Tg2a cells versus undifferentiated controls (ctl; gray). Differentiation was triggered for three days with three independent protocols: LIF withdrawal from FCS/LIF cultures (no LIF; red), 2i and LIF withdrawal from 2i+LIF cultures (N2B27; blue) or EpiLC differentiation from 2i+LIF cultures (EpiLC; purple). Values were normalized to $T b p$ and fold changes calculated to their respective control culture. Each dot represents an independent replicate and the bar and error bars the corresponding means and standard errors. (B) RT-qPCR analysis of Suv39h1as (red) and Suv39h1 (blue) expression upon OCT4 depletion for the indicated time (X-axis). Values were normalized to Tbp. Each dot represents an independent replicate and the line the corresponding mean with standard errors. (C) Representative sm-FISH of Suv39h1as and Suv39h1 RNA molecules, followed by DNA-FISH visualising the Suv39h1/Suv39h1as locus in E14Tg2a cells $(n=358)$. Selected loci transcribing either Suv39h1, Suv39h1as or both genes are indicated with arrow heads: blue, Suv39h1-only (30\%); red, Suv39h1as-only (30\%); yellow for cells transcribing both (20\%). (D) Boxplots (median; $25-75 \%$ percentiles; error bars) showing the number of Suv39h1 diffusible molecules counted in cells presenting an active (TS+) or inactive (TS-) Suv39h1as gene, counted in 358 E14Tg2a cells.

summary, both smFISH and RT-qPCR experiments showed that Suv39hl and Suv39hlas display anticorrelated expression patterns. This anticorrelation stems from single cell dynamics where the transcription of the antisense leads to a slight reduction of the transcriptional frequency of Suv39hl and, more significantly, to reduced expression of diffusing mRNAs. These observations indicate that Suv39hlas acts as a repressor of Suv39hl. Since Suv39hlas is downregulated upon differentiation and upon the loss of OCT4, our data suggests that pluripotent TFs activate Suv39hlas transcription which, in turn, downregulates Suv39hl expression.

\section{A simple OCT4-Suv39h1as-Suv39h1 circuitry couples H3K9 methylation to pluripotency.}

To functionally establish the relationships between OCT4, Suv39hlas, Suv39hl and H3K9 methylation, we designed two gRNAs to delete $5,5 \mathrm{~kb}$ encompassing the two promot-

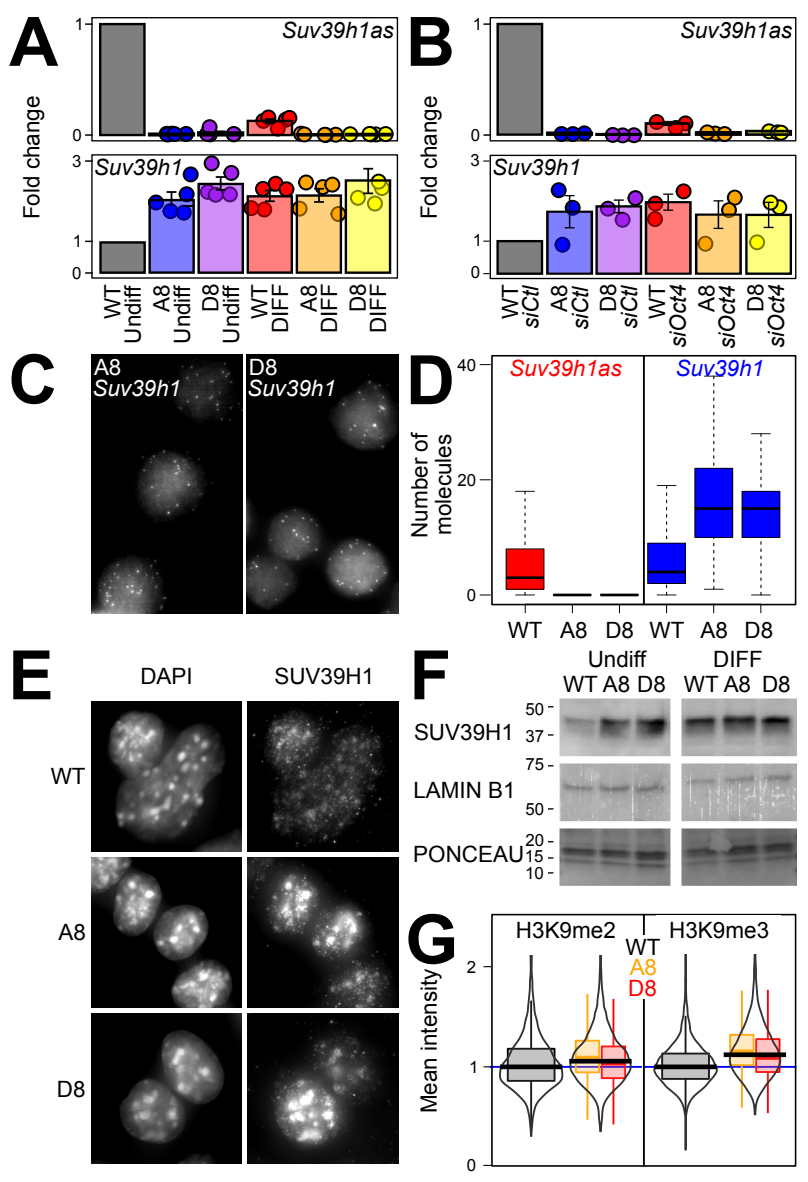

Fig. 4. OCT4 represses Suv39h1 via Suv39h1as to reduce H3K9 methylation in ES cells. (A) Expression fold change of Suv39h1as (top) and Suv39h1 (bottom) in WT (E14Tg2a) and Suv39h1as-mutant cells (A8 and D8) cultured in undifferentiated (FCS/LIF) or differentiating conditions ( 3 days without LIF). Values were normalized to Tbp. Each dot represents an independent replicate and the bar and error bars the corresponding means and standard errors. (B) Expression fold change of Suv39h1as (top) and Suv39h1 (bottom) in wild-type (WT - E14Tg2a) and Suv39h1as-mutant cells (A8 and D8) knocked-down with either control or Oct4targetted siRNAs. Values were normalized to Tbp. Each dot represents an independent replicate and the bar and error bars the corresponding means and standard errors. (C) Representative sm-FISH images of Suv39h1 in A8 and D8 cells (wild-type cells presented in Fig.3C). (D) Boxplots (median; 25-75\% percentiles; error bars) showing the number of Suv39h1as (red) or Suv39h1 (blue) diffusible molecules counted in wild-type $(\mathrm{WT} ; \mathrm{n}=358$ ) or mutant cells $(A 8, \mathrm{n}=289$; D8, $n=$ 270). (E) Representative SUV39H1 immunofluorescence of wild-type (E14Tg2a) and Suv39h1-mutant ES cells (A8 and D8). (F) Representative Western-Blot of SUV39H1, LAMIN B1 and corresponding Ponceau for wild-type (E14Tg2) and mutant cells (A8 and D8) in undifferentiated (FCS/L) and differentiating (3 days without LIF) conditions. On the left of each image is indicated protein scale in $\mathrm{kDa}$. (G) Violin and boxplots (median; $25-75 \%$ percentiles; error bars) presenting relative $\mathrm{H} 3 \mathrm{~K} 9 \mathrm{me} 2$ (left) and $\mathrm{H} 3 \mathrm{~K} 9 \mathrm{me} 3$ (right) mean intensity values of E14Tg2a (WT - black; $n=12881$ for H3K9me2 and $n=12053$ for H3K9me3) or mutant cells (A8, yellow, $n=3553$ cells for $\mathrm{H} 3 \mathrm{~K} 9$ me2 and 2081 for $\mathrm{H} 3 \mathrm{~K} 9$ me3; D8, red, $\mathrm{n}=5050$ cells for H3K9me2 and 2641 for $\mathrm{H} 3 \mathrm{~K} 9 \mathrm{me} 3$ ), assessed by immunofluorescence.

ers of Suv39hlas. Two independent KO clones, A8 and D8, were generated. PCR genotyping confirmed the deletion and analysis of their karyotypes did not reveal any overt defect. Moreover, RT-qPCR showed a complete loss of Suv39hlas expression (Fig.4A). We then addressed the impact of Suv39hlas depletion on Suv39hl expression, both before and during differentiation. In the two mutant clones we observed an increase of Suv39hl expression in undifferentiated cells ( $\mathrm{p}=0.011$ and 0.0016 for $\mathrm{A} 8$ and $\mathrm{D} 8$, respectively), reaching the levels observed upon differentiation in WT cells 
(Fig.4A). In differentiating cells, when Suv39hlas is naturally silenced, the deletion had no impact (Fig.4A), as expected, regardless of the differentiation protocol (Fig.S1D). Therefore, these results indicate that Suv39hlas acts as a pluripotency-associated repressor of Suv39hl expression. However, this does not imply that OCT4 exclusively represses Suv39h1 expression via Suv39hlas. To address this, we used siRNAs targeting Oct4 to test whether in the absence of Suv39hlas, the loss of OCT4 would lead to any modification of Suv39hl expression. Whereas in wild-type cells the knock-down of Oct4 (above 80\% efficiency, Fig.S1E), led to higher ( $\mathrm{p}=0.027$ ) Suv39hl expression (Fig.4B), as expected, in mutant cells it was fully inconsequent (Fig.4B). This indicates that the OCT4-dependent repression of Suv39h1 is exclusively mediated by Suv39hlas. Next, we performed smFISH to study Suv39hl upregulation with single cell resolution (Fig.4C). We observed a marked increase in the transcriptional frequency of Suv39hl, rising from $47.6 \%$ in WT to $76.3 \%$ and $74.1 \%$ in $\mathrm{A} 8$ and D8, respectively ( $\mathrm{p}<10^{-10}$ for both clones). Moreover, the number of Suv39h1 mRNAs per cell also increased substantially $\left(\mathrm{p}<10^{-15}\right)$, with virtually no cell displaying an absence of Suv39h1 mRNAs (Fig.4D). This increase in Suv39hl expression was accompanied by higher levels of SUV39H1 protein expression and accumulation at heterochromatic regions such as chromocenters, as established by immuno-staining and western-blot (Fig.4E,F; Fig.S1F). Consequently, in both mutant clones we observed higher levels of $\mathrm{H} 3 \mathrm{~K} 9 \mathrm{me} 2$ and $\mathrm{H} 3 \mathrm{~K} 9 \mathrm{me} 3\left(\mathrm{p}<10^{-8}\right.$ for both marks and cell clones), establishing a direct link between Suv39hlas and the global levels of H3K9 methylation in ES cells (Fig.4G).

\section{Suv39h1as modifies the chromatin of the} Suv39h1as/Suv39h1 locus.

We have observed that in mutant ES cells lacking Suv39hlas expression, the transcriptional frequency of Suv39hl increases from 50 to $75 \%$. Moreover, the absence of Suv39hlas is not accompanied by increased stability of Suv39hl mRNAs (Fig.S1G). Therefore, Suv39hlas is likely to act as a transcriptional repressor of Suv39hl. To explore this, and given that other antisense transcription units have been shown to modify the chromatin of their corresponding sense gene ${ }^{29-31}$, we used a ChIP approach to establish the histone modification profiles of the locus (Fig.5). First, we monitored H3K4 methylation profiles. We found H3K4me1 and me2, which usually mark transcriptionally competent regions ${ }^{32}$, to globally decorate the locus with minimal focal accumulation at the promoters. Conversely, H3K4me3, a mark of activity ${ }^{32}$, was focally enriched at the Suv39hl promoter and displayed low levels over the antisense promoter. We then profiled the active histone acetylation marks, H3K9ac and H3K27ac. Similarly to $\mathrm{H} 3 \mathrm{~K} 4 \mathrm{me} 3$, we found $\mathrm{H} 3 \mathrm{~K} 9 \mathrm{ac}$ to preferentially mark the Suv39hl promoter. In contrast, both sense and antisense gene promoters where enriched for $\mathrm{H} 3 \mathrm{~K} 27 \mathrm{ac}$. In mutant cells we observed a global decrease of $\mathrm{H} 3 \mathrm{~K} 4 \mathrm{me} 1 / \mathrm{me} 2$ over the region transcribed by Suv39hlas $\left(\mathrm{p}<10^{-3}\right)$, particularly before it reaches the Suv39hl gene body (Fig.5), indicat-

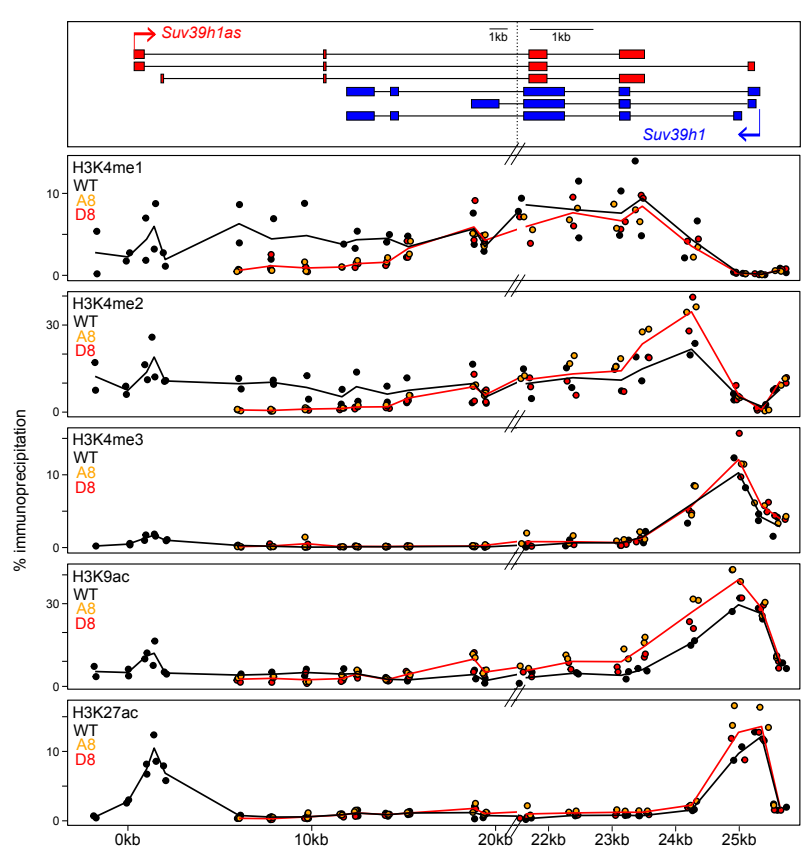

Fig. 5. Suv39h1as triggers complex chromatin changes across the locus. Chromatin immunoprecipitation profile (Y-axis, percentage of immunoprecipitation) of H3K4me1, H3K4me2, H3K4me3, H3K9ac and H3K27ac, as indicated, across Suv39h1/Suv39h1as locus in wild-type (E14Tg2a, black) and Suv39h1as-mutant cells (A8, yellow dots; D8, red dots; the red line represents the average of all data points for mutant clones). The $\mathrm{X}$-axis represents genomic distances in $\mathrm{kb}$ with respect to the Suv39h1as transcription start site, as schematized on top. Note a break on the scale of the genomic coordinates at around $X=21 \mathrm{~kb}$.

ing its transcription promotes the establishment of these marks. The lack of $\mathrm{H} 3 \mathrm{~K} 4 \mathrm{me} 1 / \mathrm{me} 2$ reduction within the region transcribed by both genes suggests that the increased transcription of Suv39hl may have a compensatory role. Moreover, H3K4me2, H3K9ac and H3K27ac, all marks of gene activity, showed a slight but statistically significant increase $(\mathrm{p}<0.05)$ at Suv39hl promoter in the absence of Suv39hlas (Fig.5). Altogether, this analysis suggests that the loss of Suv39hlas leads to increased Suv39hl transcription at least in part mediated by increased euchromatinisation of the Suvar39h1 promoter. These results suggest a simple mechanism where Suv39hlas modulates the local chromatin environment of Suv39hl to decrease the probability of its transcription, leading to a global lower level of Suv39hl mRNA and, subsequently, protein.

\section{The lack of Suv39h1as leads to accelerated differentiation commitment.}

We finally wondered whether the increase of H3K9 methylation taking place in Suv39hlas mutant cells had any physiological impact. First, we used clonal assays to assess self-renewal and differentiation efficiency (Fig.6A,B). Either in conditions of reinforced self-renewal (2i/LIF), in traditional serum-containing culture medium (FCS/LIF) or in the absence of LIF (FCS), the number of alkaline-phosphatase colonies, a marker of pluripotent cells, was identical between wild-type and mutant clones. Hence, the presence of increased H3K9 methylation is largely inconsequential for self-renewal and for the loss of pluripotency. In agreement, both wild-type and mutant cells proliferate and differenti- 


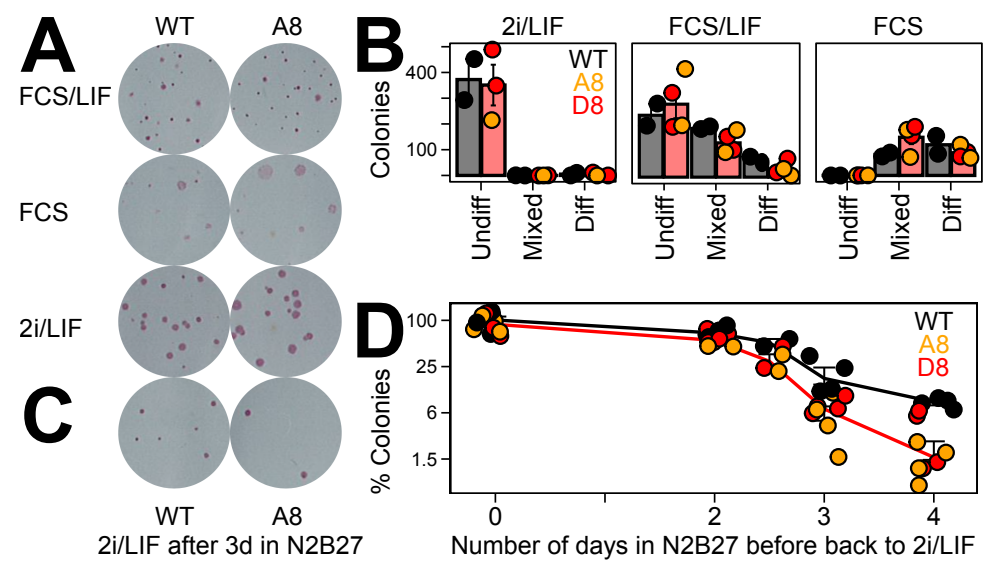

Fig. 6. Accelerated commitment into differentiation in the absence of Suv39h1as. (A) Representative alkaline-phosphatase staining of ES cell colonies cultured as indicated. (B) Number of wild-type (E14Tg2a, black) or Suv39h1as-mutant (A8, orange points; D8, red points) colonies characterized as undifferentiated, mixed or differentiated after culturing them as indicated. Each dot represents an independent replicate and the bar and error bars the corresponding means (black for wild-type and red for the mean of all data points for mutant clones) and standard errors. (C) Alkaline-phosphatase staining of ES cell colonies cultured in 2i/LIF after 3 days in N2B27 for wild-type (E14Tg2a) and Suv39h1as-mutant cells (A8). (D) Percentage of alkaline-phosphatase positive colonies cultured in 2i/LIF after differentiating them for the indicated number of days (Xaxis) in N2B27. D0, undifferentiated cells were set as $100 \%$. Each dot represents an independent replicate (wild-type, black; Suv39h1as-mutant clones in orange, A8, and red, D8) and the line the corresponding mean and standard error (all mutant datapoints were averaged to obtain the red line).

ate normally, as evaluated morphologically (Fig.S1H) and by marker expression (Fig.S1I). However, during differentiation, the role of $\mathrm{H} 3 \mathrm{~K} 9$ methylation is to restrict cell fate and developmental competence ${ }^{\mathbf{1 8 , 3 3}}$, more than to elicit differentiation. Therefore, we reasoned that the loss of Suv39hlas could modulate the timing of commitment into differentiation. To test this, we used an established assay ${ }^{\mathbf{3 4}}$ whereby wild-type and mutant clones were differentiated in parallel and, every day, the cells were harvested and reseeded clonally in 2i/LIF: only those cells that were not yet committed into irreversible differentiation can self-renew and form undifferentiated colonies (Fig.6C,D). As previously shown, we observed that commitment took place between days 2 and 3 in wild-type cells, with a reduction in clonogenicity of nearly 90\% (Fig.6D). In mutant cells, however, the reduction in the number of undifferentiated colonies was more marked from day 2.5 onwards (Fig.6D; $\mathrm{p}=0.0666,0.0020$ and 0.0041 for days $2.5,3$ and 4 , respectively). Therefore, the premature establishment of higher levels of $\mathrm{H} 3 \mathrm{~K} 9 \mathrm{me} 2 / \mathrm{me} 3$ in ES cells facilitates the irreversible commitment into differentiation, in line with the role of these repressive marks in locking cell fate changes ${ }^{\mathbf{2 0}}$.

\section{Discussion}

In this study, we have identified a genetic network linking the control of the global levels of H3K9 methylation to pluripotency. The pluripotency network, mainly through OCT4, activates an antisense lncRNA to the Suv39h1 gene (Suv39hlas), repressing Suv39hl expression. Consequently, the level of H3K9 methylation is reduced. Upon differentiation, the collapse of the pluripotency network leads to the silencing on Suv39hlas, enabling increased SUV39H1 expression and $\mathrm{H} 3 \mathrm{~K} 9$ methylation, which controls the timing and efficiency of the irreversible commitment into differentiation. This genetic setup may act as a time-delay generator, enabling ES cells to filter out transient fluctuations in the activity of the pluripotency network ${ }^{35}$ : only a long decrease in activity of the pluripotency network may be sufficient to elicit the increase in SUV39H1 expression that will follow the extinction of Suv39hlas.

Antisense lncRNAs are frequent in mammals, with $29 \%$ of canonical protein coding genes displaying antisense transcription ${ }^{36}$. Given their antisense orientation and the resulting complementarity, antisense lncRNAs can theoretically regulate their cis-linked sense gene through a wide variety of mechanisms. By deleting the Suv39hlas promoter region, we found that Suv39hlas controls the frequency of Suv39hl transcription and has no impact on Suv39h1 mRNA stability. However, the exact mechanisms by which Suv39hlas represses Suv39hl transcription remain open, even though we show it has complex influences on the chromatin. On the one hand, Suv39hlas triggers H3K4me1 and me 2 throughout the locus; on the other, it slightly reduces H3K4me2, H3K4me3, H3K9ac and H3K27ac at the Suv39hl promoter. Notably, these effects are reminiscent of those associated with the regulation of Xist by its antisense $T$ si $x^{29-31}$, possibly revealing a general property of antisense transcription. While it is possible that the slight increase of euchromatic marks at the Suv39hl promoter are sufficient to increase the frequency of Suv39hl transcription, other potential mechanisms cannot be excluded. For instance, the antisense/sense polymerases could enter into physical collision and transcriptional abortion ${ }^{37}$, modulating Suv39hl elongation. Moreover, Suv39hlas presents two exons nearly fully overlapping $S u v 39 h 1$ exons, a gene structure that is conserved at the human Suv39h1as/Suv39h1 locus (Fig.S1J). Therefore, while the transcriptional effects are clear, Suv39hlas may deploy additional post-transcriptional mechanisms to downregulate Suv39hl mRNA expression, such as masking splice sites as it has been reported for $E r b A a^{38}$. Finally, other mechanisms fully independent of Suv39hlas transcription or of the produced lncRNA cannot be excluded, such as direct competition between the sense and antisense promoters ${ }^{39}$.

The deletion of Suv39hlas promoter and the ensuing increase in $\mathrm{H} 3 \mathrm{~K} 9$ methylation, appears to be largely inconsequent for ES cells: they self-renew and differentiate efficiently. This observation is in line with others, where histone modifiers have been either invalidated or ectopically expressed in ES cells with minor consequences for selfrenewal ${ }^{40,41}$. However, despite the fact that Suv39hlas mutant cells self-renew and differentiate normally, we asked whether the timing of commitment into differentiation is altered. Our results showed a faster and more efficient com- 
mitment into differentiation, suggesting that the global levels of $\mathrm{H} 3 \mathrm{~K} 9$ methylation contribute to irreversibly lock the loss of pluripotency. This observation adds to the notion of $\mathrm{H} 3 \mathrm{~K} 9$ methylation acting as an epigenetic barrier providing robustness to cell fate changes ${ }^{33}$. Moreover, our results also underscore the dominance of pluripotency TFs over chromatin modifications ${ }^{41}$. We had already shown that NANOG, another key pluripotency TF, controls H3K27me3 levels, particularly during early differentiation ${ }^{22}$. Here, we complement this notion with OCT4 controlling H3K9me3 via the Suv39hlas/Suv39hl tandem. Together, these results place the control of global levels of heterochromatin marks under the activity of the pluripotency network, extending the concept of the genetic dominance of pluripotency. Whether our observations and conclusions can be extended to early mouse embryogenesis and to the acquisition and loss of pluripotency in vivo is now a question of primary importance. Notably, H3K9 methylation levels are exquisitely regulated during early embryogenesis ${ }^{\mathbf{4 2}}$. It is noteworthy that SUV39H1 is absent in oocytes and its expression starts at the 2-4 cell transition stage ${ }^{\mathbf{4 3}}$, when the reconfiguration of constitutive heterochromatin as chromocenters is initiated. Moreover, the overexpression of SUV39H1 during the early stages of embryogenesis leads to developmental defects. Indeed, using different strategies, Zhang et al. ${ }^{\mathbf{1 3}}$ and Burton et al. ${ }^{\mathbf{1 4}}$ overexpressed SUV39H1 in the zygote and observed an increase of $\mathrm{H} 3 \mathrm{~K} 9 \mathrm{me} 3$ levels leading to early developmental arrest at the time of compaction. Therefore, an important hypothesis emerges from this work: Suv39hlas could be a key regulator of Suv39hl during early embryogenesis, holding its expression until the appropriate time to enable the timely establishing of the first heterochromatic structures in the embryo.

Supplementary information One supplementary figure accompanies this manuscript, it can be found at the end of this document. Two Supplementary Tables and Methods are available online.

Acknowledgements. This study was conceived by P.N with inputs from L.B. and V.H.. Experiments were designed and executed by L.B., with help from A.D., V.H., A.T., S.V-P. and M.C-T. RNA-seq analyses and assembly were done by N.G., N.O., A.C. and I.U. The paper was written by L.B. and P.N. L.B. acknowledges the Ecole Normale Supérieure and Sorbonne Université for funding. P.N. and M.C-T. acknowledge the Labex Revive (Investissement d'Avenir; ANR-10-LABX-73), the Institut Pasteur and the CNRS for funding.

Declaration of interests. The authors declare no competing interests.

\section{References}

1. Li E. Chromatin modifications and epigenetic reprogramming in mammalian development. Nat Rev Genet 3, 662-673 (2002).

2. Allshire, R. C. Madhani, H. D. Ten principles of heterochromatin formation and function. Nat Rev Mol Cell Biol 19, 229-244 (2018).

3. Trojer, P. Reinberg, D. Facultative Heterochromatin: Is There a Distinctive Molecular Signature? Molecular Cell 28, 1-13 (2007).
4. Saksouk, N., Simboeck, E. Déjardin, J. Constitutive heterochromatin formation and transcription in mammals. Epigenetics Chromatin 8, 3 (2015).

5. Liu, J., Ali, M. Zhou, Q. Establishment and evolution of heterochromatin. Annals of the New York Academy of Sciences 1476, 59-77 (2020).

6. Tosolini, M. et al. Contrasting epigenetic states of heterochromatin in the different types of mouse pluripotent stem cells. Scientific Reports 8, 5776 (2018).

7. Walter, M., Teissandier, A., Pérez-Palacios, R. Bourc'his, D. An epigenetic switch ensures transposon repression upon dynamic loss of DNA methylation in embryonic stem cells. Elife 5, (2016).

8. Johnson, W. L. et al. RNA-dependent stabilization of SUV39H1 at constitutive heterochromatin. eLife 6, e25299 (2017).

9. Nicetto, D. and Zaret, K. Role of H3K9me3 heterochromatin in cell identity establishment and maintenance. Curr Opin Genet Dev, 55, 1-10 (2019).

10. Janssen, A., Colmenares, S. U. Karpen, G. H. Heterochromatin: Guardian of the Genome. Annu. Rev. Cell Dev. Biol. 34, 265-288 (2018).

11. Dodge, J. E., Kang, Y.-K., Beppu, H., Lei, H. Li, E. Histone H3-K9 Methyltransferase ESET Is Essential for Early Development. MCB 24, 2478-2486 (2004).

12. Tachibana, M. et al. G9a histone methyltransferase plays a dominant role in euchromatic histone H3 lysine 9 methylation and is essential for early embryogenesis. Genes Dev 16, 1779-1791 (2002).

13. Zhang, Y.-L. et al. DCAF13 promotes pluripotency by negatively regulating SUV39H1 stability during early embryonic development. EMBO J. 37, (2018).

14. Burton, A. et al. Heterochromatin establishment during early mammalian development is regulated by pericentromeric RNA and characterized by non-repressive H3K9me3. Nat Cell Biol 22, 767-778 (2020).

15. Bernstein, B. E. et al. A bivalent chromatin structure marks key developmental genes in embryonic stem cells. Cell 125, 315-326 (2006).

16. Mikkelsen, T. S. et al. Genome-wide maps of chromatin state in pluripotent and lineage-committed cells. Nature 448, 553-560 (2007).

17. Wen, B., Wu, H., Shinkai, Y., Irizarry, R. A. Feinberg, A. P. Large histone H3 lysine 9 dimethylated chromatin blocks distinguish differentiated from embryonic stem cells. Nat Genet 41, 246-250 (2009).

18. Sridharan, R. et al. Proteomic and genomic approaches reveal critical functions of H3K9 methylation and heterochromatin protein- $1 \gamma$ in reprogramming to pluripotency. Nat Cell Biol 15, 872-882 (2013).

19. Wei, J. et al. KDM4B-mediated reduction of H3K9me3 and H3K36me3 levels improves somatic cell reprogramming into pluripotency. Sci Rep 7, 7514 (2017).

20. Becker, J. S., Nicetto, D. Zaret, K. S. H3K9me3Dependent Heterochromatin: Barrier to Cell Fate Changes. Trends in Genetics 32, 29-41 (2016).

21. Staněk, D. Long non-coding RNAs and splicing. 
Essays Biochem EBC20200087 (2021).

22. Heurtier, V. et al. The molecular logic of Nanoginduced self-renewal in mouse embryonic stem cells. Nat Commun 10, 1109 (2019).

23. Festuccia, N. et al. Esrrb Is a Direct Nanog Target Gene that Can Substitute for Nanog Function in Pluripotent Cells. Cell Stem Cell 11, 477-490 (2012).

24. Niwa, H., Miyazaki, J. Smith, A. G. Quantitative expression of Oct-3/4 defines differentiation, dedifferentiation or self-renewal of ES cells. Nat. Genet. 24, 372-376 (2000).

25. Navarro, P. et al. OCT4/SOX2-independent Nanog autorepression modulates heterogeneous Nanog gene expression in mouse ES cells. EMBO Journal 31, 4547-4562 (2012).

26. Festuccia, N. et al. Transcription factor activity and nucleosome organization in mitosis. Genome Research 29, 250-260 (2019).

27. Wang, L. et al. CPAT: Coding-Potential Assessment Tool using an alignment-free logistic regression model. Nucleic Acids Res 41, e74 (2013).

28. Kang, Y.-J. et al. CPC2: a fast and accurate coding potential calculator based on sequence intrinsic features. Nucleic Acids Res 45, W12-W16 (2017).

29. Ohhata, T. et al. Dynamics of transcription-mediated conversion from euchromatin to facultative heterochromatin at the Xist promoter by Tsix. Cell Reports 34, 108912 (2021).

30. Navarro, P., Pichard, S., Ciaudo, C., Avner, P. Rougeulle, C. Tsix transcription across the Xist gene alters chromatin conformation without affecting Xist transcription: implications for X-chromosome inactivation. Genes Dev. 19, 1474-1484 (2005).

31. Navarro, P., Page, D. R., Avner, P. Rougeulle, C. Tsix-mediated epigenetic switch of a CTCF-flanked region of the Xist promoter determines the Xist transcription program. Genes Development 20, 2787-2792 (2006).

32. Santos-Rosa, H. et al. Active genes are tri-methylated at K4 of histone H3. Nature 419, 407-411 (2002).

33. Wang, C. et al. Reprogramming of H3K9me3dependent heterochromatin during mammalian embryo development. Nat Cell Biol 20, 620-631 (2018).

34. Kalkan, T. et al. Tracking the embryonic stem cell transition from ground state pluripotency. Development 144, 1221-1234 (2017).

35. Torres-Padilla, M.-E. Chambers, I. Transcription factor heterogeneity in pluripotent stem cells: a stochastic advantage. Development 141, 2173-2181 (2014).

36. Katayama, S. et al. Antisense transcription in the mammalian transcriptome. Science 309, 1564-1566 (2005).

37. Prescott, E. M. Proudfoot, N. J. Transcriptional collision between convergent genes in budding yeast. Proc Natl Acad Sci U S A 99, 8796-8801 (2002).

38. Hastings, M. L., Milcarek, C., Martincic, K., Peterson, M. L. Munroe, S. H. Expression of the thyroid hormone receptor gene, erbAalpha, in B lymphocytes: alternative mRNA processing is independent of differentiation but correlates with antisense RNA levels. Nucleic Acids Res 25,
4296-4300 (1997).

39. Cho, S.W. et al. Promoter of lncRNA Gene PVT1 Is a Tumor-Suppressor DNA Boundary Element. Cell. 173(6):1398-1412.e22 (2018).

40. Peters, A. H. F. M. et al. Loss of the Suv39h Histone Methyltransferases Impairs Mammalian Heterochromatin and Genome Stability. Cell 107, 323-337 (2001).

41. Festuccia, N., Gonzalez, I. Navarro, P. The Epigenetic Paradox of Pluripotent ES Cells. Journal of Molecular Biology 429, 1476-1503 (2017).

42. Liu, H., Kim, J.-M. Aoki, F. Regulation of histone H3 lysine 9 methylation in oocytes and early pre-implantation embryos. Development 131, 2269-2280 (2004).

43. Deng, Q., Ramskold, D., Reinius, B. Sandberg, R. Single-Cell RNA-Seq Reveals Dynamic, Random Monoallelic Gene Expression in Mammalian Cells. Science 343, 193-196 (2014). 
bioRxiv preprint doi: https://doi.org/10.1101/2021.09.07.459323; this version posted September 7, 2021. The copyright holder for this preprint (which was not certified by peer review) is the author/funder, who has granted bioRxiv a license to display the preprint in perpetuity. It is made available under aCC-BY-NC-ND 4.0 International license.
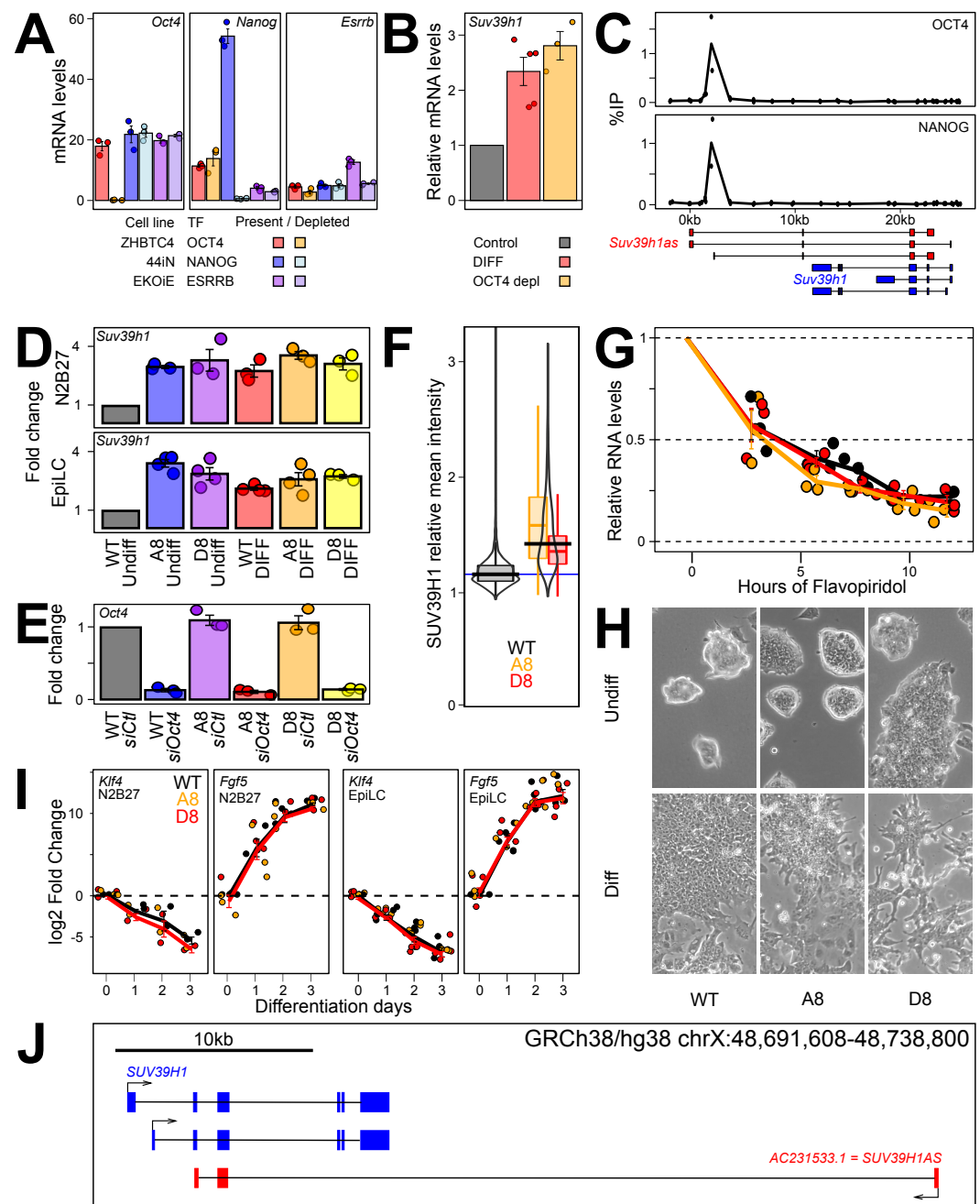

GRCh38/hg38 chrX:48,691,608-48,738,800

1

AC231533.1 = SUV39H1AS

Supplementary Information, Fig. S 1. Additional information on Suv39h1 regulation by OCT4 and Suv39h1as. (A) Expression of Oct4, Nanog or Esrrb upon inducing their depletion in specific dox-inducible knock-out lines. Note in EKOiE the remnant expression of Esrrb produces a truncated, non-functional protein ${ }^{23}$. Each dot represents an independent replicate and the bar the corresponding mean and standard error. (B) RT-qPCR validation of Suv39h1 overexpression upon differentiation (DIFF, 3 days without LIF, red) or upon OCT4 depletion (24h, orange). Each dot represents an independent replicate and the histogram the corresponding mean and standard error. (C) ChIP-qPCR validation of OCT4 and NANOG binding at the promoter region of Suv39h1as in E14Tg2a (X-axis represents genomic distances in kb with respect to the Suv39h1as transcription start site). Each black dot represents the percentage of immunoprecipitation (\%IP; Y-axis) of an independent replicate and the line the corresponding mean. A schematic representation of the locus with different isoforms is shown below. (D) RT-qPCR confirmation of lack of increased Suv39h1 upregulation in differentiated Suv39h1as-mutant cells obtained by N2B27 or EpiLC assays as compared to wild-type cells. Each dot represents an independent replicate and the histogram the corresponding mean and standard error. (E) RT-qPCR confirmation of Oct4 knock-down in wild-type and Suv39h1as-mutant cells. Each dot represents an independent replicate and the histogram the corresponding mean and standard error. (F) Violin and box-plots (median; $25-75 \%$ percentiles; error bars) showing immunofluorescence quantification of SUV39H1 mean intensity in wild-type (black; $n=4048$ cells) and mutant cells (A8 - yellow; $n=4949$ cells and D8 - red; $n=4448$ cells). (G) Analysis of Suv39h1 mRNA half-life in wild-type and Suv39h1as-mutant cells, performed and presented as in Fig. 2A. (H) Representative bright-field photomicrographs of wild-type (E14Tg2a) and mutant (A8 and D8) cells cultured in undifferentiating (FCS/LIF) and differentiating (3 days of LIF withdrawal) conditions. (I) RT-qPCR analysis of ES (KIf4) and differentiation (Fgf5) markers during differentiation in N2B27 or in EpiLC conditions, as indicated, in wild-type (E14Tg2A, black) and in Suv39h1as-mutant cells (A8, orange, and D8, red). Each dot represents an independent replicate and the lines corresponding means (Black for WT and red for both mutant) with error bars. (J) Schematic representation of the SUV39H1/SUV39H1as locus in the human genome (Gencode V36 assembly). 